\title{
INFLUENCIAS OLÍMPICAS MODERNAS: OLIMPISMO PARA EL DESARROLLO Y PAZ EN GUATEMALA
}

\author{
Pedro Danilo Ponciano Nuñez \\ Candidato a doctor Universidad de Vigo \\ Director y docente en Universidad del Valle (Guatemala) \\ pdponciano@uvg.edu.gt
}

Fecha de recepción: julio 2021

Fecha aceptación: noviembre 2021

http://doi.org/10.15366/citius2021.14.2.002

\section{Resumen:}

El objeto de este estudio de investigación es doble. Primero, explicar un marco histórico sobre la práctica de la actividad física desde la antigüedad hasta los tiempos modernos donde se integra la promoción del cambio social y el desarrollo sostenible. En segundo lugar, mostrar un panorama de iniciativas sociales que utilizan la educación física y el deporte en Guatemala, que involucran al movimiento olímpico dentro de sus intervenciones sociales. El estudio se llevó a cabo mediante una exhaustiva metodología de investigación documental dentro de bases de datos de artículos científicos especializados. Además, el uso de literatura de estudios olímpicos e iniciativas deportivas sociales seleccionadas. Los resultados muestran cómo el Olimpismo y el deporte se integran en el trabajo prosocial hacia la promoción de objetivos de desarrollo sostenible y proponen futuras iniciativas para el desarrollo de esta línea de investigación limitada en Guatemala.

Palabras clave: Olimpismo en Guatemala, Cambio Social, Teoría del Deporte para el Desarrollo, Olimpismo en Acción, Educación Física

Title: MODERN OLYMPIC INFLUENCES: OLYMPISM FOR DEVELOPMENT AND PEACE IN GUATEMALA

\begin{abstract}
:
The object of this research study is twofold. First, explain a historical framework on the practice of physical activity from ancient times to modern times where the promotion of social change and sustainable development is integrated. Second, to show a panorama of social initiatives that use physical activity in Guatemala, that involve the Olympic movement within their social interventions. The study was carried out through an exhaustive documentary research methodology within databases of specialized scientific articles. In addition, the use of literature from Olympic studies and selected social sports initiatives. The results show how Olympism and sport are integrated into the pro-social work towards the promotion of sustainable development objectives and propose future initiatives for the development of this line of research limited in Guatemala.
\end{abstract}

Keywords: Olympism in Guatemala, Social Change, Sport for Development Theory, Olympism in Action, Physical Education

\section{INTRODUCCIÓN}

Los últimos veinte años, múltiples organizaciones alrededor del mundo como el Comité Olímpico Internacional (COI) y la Organización de Naciones Unidas (ONU) han unido esfuerzos sistemáticos hacia la promoción de los objetivos de desarrollo sostenible y construcción de la paz a través del Movimiento Olímpico y del uso deporte para impactar a nivel global en la sociedad 
(Ponciano y Lyras, 2018). Sin embargo, ante la desaparición de la oficina de Deporte para el Desarrollo y la Paz de la ONU en el año 2017 se provocó dudas sobre el futuro de dicho sector. En este sentido, a pesar de dicha desaparición ha sido la relación del COI y la ONU a través del tiempo que ha permitido mantenerse firmes hacia dicho objetivo a través de la resolución «Construir un mundo pacífico y mejor a través del deporte y el ideal olímpico» (COI 2000). Por lo tanto, el ideal olímpico y el deporte es considerada una plataforma sostenible para la promoción de objetivos sociales más amplios como la educación, salud, construcción de paz, la igualdad de género, entre otros.

De Coubertin describe el concepto de ideal olímpico como el ascenso de la mente y el alma que se convierte en un medio de contribución para superar barreras entre naciones y culturas. Por ejemplo, esta filosofía es hallada en el modelo educativo del gimnasio practicado por los Atenienses que enfatizaba la necesidad de desarrollar de manera equilibrada el cuerpo y la mente como componentes fundamentales en su sistema educativo (Clastres, 2010; Sakoulas, 2016). Esta idea filosófica ha sido interpretada bajo el concepto de olimpismo, refiriéndose al uso del deporte para promover el desarrollo equilibrado de las personas, con la finalidad de colaborar en la construcción de sociedades más pacíficas y hacia el desarrollo humano (McLaughlin y Torres, 2012). Sin embargo, este concepto no es novedoso, ya que en la antigua civilización Helénica las competencias atléticas eran consideradas un medio de formación integral de personas útiles para sus comunidades y capaces de participar en los Juegos Olímpicos (Naul et al., 2017). Además, la mitología griega refiere que los Juegos Olímpicos antiguos respondían al interés de detener las guerras, conflictos entre ciudades del mundo griego y de esta manera disminuir el odio o agresión al servir como un medio de coexistencia pacífica entre grupos (Lyras, 2021).

Los antiguos Juegos Olímpicos fueron establecidos como más que una competencia atlética, al combinar ideales educativos con actividades culturales o religiosas, que posteriormente serían los elementos ceremoniales de los Juegos Olímpicos modernos (Koulouri, 2006). Estos elementos serian recuperados en la historia a pesar de la abolición de los juegos por ser considerado un evento pagano y de culto al cuerpo lo que limitó su continuidad por cierto tiempo hasta el renacimiento moderno de los mismos (Koulouri, 2006). No obstante, ha sido posterior al renacimiento de los Juegos Olímpicos modernos por De Coubertin por medio de sus escritos y han sido reconocidos estos conceptos de los antiguos juegos helénicos, los cuales fueron implementados dentro del curriculum educativo en Francia. Al mismo tiempo, esto evidencia que los juegos antiguos eran un medio dirigido hacia la innovación y el progreso social de la civilización de la antigua Grecia (Teetzel, 2012).

\section{JUSTIFICACIÓN}

Tomando en consideración las lógicas y estructuras históricas arriba desarrolladas, se ilumina la relación antigua y moderna en torno al olimpismo percibido como un vehículo de transformación social y como se integra su esencia a la nueva tendencia popular del deporte para el desarrollo (DPD). La definición de DPD ampliamente reconocida en el cuerpo del conocimiento fue establecida por Lyras y Welty Peachey (Lyras y Welty Peachey, 2011, p. 311): 
«El uso del deporte para ejercer una influencia positiva en la salud pública, la socialización de niños, jóvenes y adultos, la inclusión social de personas desfavorecidas, el desarrollo económico de regiones y estados, y el fomento del intercambio intercultural y la resolución de conflictos.»

Esta definición, aunque es establecida hasta el año 2011 por sus autores, es resultado del amplio interés de los investigadores en esta área del conocimiento a partir de la resolución «Construir un mundo pacífico y mejor a través del deporte y el ideal olímpico» (COI, 2000). Por lo tanto, a partir de ese momento ha aumentado paulatinamente la investigación en este ámbito a través de los años alrededor del mundo; sin embargo, sigue siendo limitado en América Latina (Schulenkorf, Sherry, y Rowe, 2016). Desde la experiencia del autor, esto puede ser consecuencia de la falta de habilidad de los investigadores locales — en muchos de los casosdel dominio del idioma inglés, lo cual imposibilita publicar los resultados de sus investigaciones en revistas consideradas de alto impacto y que tributan al DPD.

Lo anterior, nos transporta a Centro América una región con una larga historia de conflicto, desigualdad y pobreza que afecta a los y las habitantes de estos países, pero que ha sido sede de estudios recientes en el DPD. Por ejemplo, El Salvador en el año 2011, considerado uno de los países más peligrosos del mundo, se convirtió en el punto de partida para ser seleccionado como ubicación de un estudio de caso por parte de un grupo de expertos canadienses para obtener datos acerca de los resultados de una intervención basada en educación física $(\mathrm{EF})$ para la prevención de la violencia y desarrollo de habilidades para la vida (Mandigo, Corlett y Ticas, 2016). Las habilidades para la vida son destrezas que permiten a los adolescentes adquirir aptitudes necesarias para el desarrollo humano y les permiten incrementar sus posibilidades de tomar decisiones adecuadas en su vida diaria (Mandigo, Corlett y Hobin et al., 2018).

El estudio evidencia que por medio de un programa desarrollado en un ambiente seguro y permanente a través de la EF los participantes alcanzan aprendizajes significativos de sus habilidades para la vida y resolución de conflictos (Mandigo, Corlett y Hobin et al., 2018). También evidenció el bajo nivel de los modelos de aprendizaje y desarrollo de nuevos profesores, quienes aún son formados por metodologías tradicionales no contextualizadas a las realidades de los entornos del país, o que no integran herramientas para la construcción de la paz y el cambio social (Mandigo, Corlett y Hobin, et al., 2010). Por consiguiente; para convertir una escuela en una intervención social que utilice el olimpismo a través de la educación física y el deporte, es necesario hacer énfasis en el diseño curricular de las intervenciones, ya que proporciona las condiciones para crear un impacto sostenible en tiempo y espacio (Lyras y Welty Peachey, 2011).

\section{OBJETIVOS}

En Guatemala se evidencia una limitada investigación en torno al uso del olimpismo para alcanzar impactos sociales más amplios a través del deporte en las comunidades cuyas poblaciones son más vulnerables. La población guatemalteca se encuentra estratificada como en cualquier otro estado, con base a una clasificación vertical por origen: i) descendientes de la población autóctona (mayas y xincas), de los colonizadores españoles (criollos y mestizos/ladinos), de los esclavos negros (garífunas) o de emigrantes de otros países (Rato Barrio, 2009). 
En este sentido, Rato Barrio (2009) menciona que «una de las características de la población guatemalteca es la emigración, fuertemente condicionada no solo por la pobreza sino también por las situaciones de enfrentamientos entre diferentes grupos étnicos, que no han desaparecido tras los acuerdos de paz de 1996» (p.9). A causa de la emigración, la población se desplaza al extranjero a países como España, Estados Unidos y México, pero también existe el mismo fenómeno a nivel nacional del campo a la ciudad en donde las personas no encuentran una mejor situación y por el contrario, se convierten en víctimas de la delicuencia y las pandillas (maras).

A consecuencia de la violencia provocada por la delicuencia y las pandillas en diferentes partes del país, los niños, niñas, jóvenes y adultos (NNJA) en condiciones de vulnerabilidad, han encontrado limitadas sus posibilidades para hacer actividad física, ante la falta de espacios deportivos seguros a su alcance. Así mismo, se ha incrementado la presencia de organizaciones no gubernamentales (ONGs) y gubernamentales que utilizan el deporte como herramienta para enseñar valores, habilidades para la vida y en el presente estudio haremos énfasis en las organizaciones, programas y actividades cuya filosofía integra el concepto del olimpismo para la promoción del cambio social y construcción de la paz. Por lo tanto, bajo las premisas mencionadas anteriormente el estudio fue guiado por la siguiente pregunta de investigación:

¿Cuáles son las raíces e influencias olímpicas visibles en esta época en la cultura de la educación física y el deporte en Guatemala?

Los objetivos son los siguientes:

- Explicar un marco histórico sobre la práctica de la educación física y el deporte desde tiempos antiguos hasta la época moderna hacia la promoción del cambio social.

- Mostrar un panorama de iniciativas sociales que integran la filosofía del olimpismo a través de la educación física y el deporte en Guatemala.

\section{METODOLOGÍA}

El presente estudio ha utilizado técnicas de investigación de análisis documental. El análisis documental implica una serie de técnicas de búsqueda, procesamiento y almacenamiento de información contenida en documentos que de forma posterior resultan en una presentación sistemática, coherente y argumentada de un nuevo documento científico (Tancara Q, C., 1993). Por otra parte, el proceso sistemático de búsqueda fue dividido en dos fases ejecutadas durante los meses de octubre de 2019 a marzo de 2021.

En la primera fase, se llevó a cabo por medio de una revisión exhaustiva de artículos científicos en bases de datos como Google Scholars y Elsavier Scopus a través de operadores booleanos. La búsqueda permitió localizar un total de 31 artículos en revistas indexadas entre los años 1991 a 2021. Los artículos seleccionados pertenecen a las revistas siguientes: i) Journal of Sport for Development, ii) Sport Management Review, iii) Research Papers in Education, iv) International Journal of Sport and Health Science, v) Sport and Olympic-Paralympic Studies Journal, vi) European Physical Education CX Review, vii) ICHPER-SD Journal of Research, viii) Olympika: The International Journal of Olympic Studies, ix) Journal of Sports and Social 
Issues, x) Revista: Citius, Altius, Fortius y xi) Peace and Conflict: Journal of Peace Psychology.

La segunda fase, fue desarrollada durante los meses de agosto a diciembre de 2020 por medio de la determinación de documentos (libros, tesis, entre otros) que tuvieran una relación en torno al uso del olimpismo, la educación física y el deporte para alcanzar objetivos sociales más amplios. La búsqueda dio como resultado la localización de un total de 7 libros publicados entre los años 1997 a 2019. Además, se consultaron un total de 7 documentos oficiales de políticas públicas nacionales, internacionales y reportes de las iniciativas sociales seleccionadas para participar en el estudio entre los años 2000 al 2019. Finalmente, se realizó un análisis temático de los 43 recursos localizados por medio del uso de una plantilla creada a través de una hoja de software Excel 16.16.19, que permitió la construcción de un marco teórico pertinente y actualizado que permitiera dar respuesta al estudio de investigación.

\section{REVISIÓN DE LA BIBLIOGRAFÍA}

\subsection{El nacimiento del olimpismo y el renacimiento de los juegos olímpicos modernos}

El renacimiento de los Juegos Olímpicos modernos integra dos perspectivas fundamentales: i) funcionar como un proceso educativo y ii) realizar un gran evento deportivo en el que los atletas participantes del mundo compartan los valores olímpicos durante un gran festival y luego con otros atletas en sus países (Teetzel, 2012; Naul et al., 2017). Estas dos perspectivas son parte de la visión por De Coubertin, quien estuvo inspirado por los antiguos juegos promovidos en Grecia. Asimismo, su admiración por Thomas Arnold, un profesor que fue el responsable de la implementación de los programas atléticos dentro del Reino Unido y de la influencia que supuso la formación del carácter por medio del entrenamiento deportivo en Estados Unidos (Naul, et al., 2017).

Además, el renacimiento de los Juegos Olímpicos no fue obra únicamente De Coubertin, sino un esfuerzo de muchos como Evanghelos Zappas, Panagiotis Soutsos y William Penny Brookes, quienes fueron conscientes de las invaluables ideas promovidas por la antigua Grecia y específicamente de los conceptos de Kalokagathia (desarrollo armónico del cuerpo y la mente) y el Aréte (excelencia) que estuvieron presentes en los tiempos antiguos (Teetzel, 2012).

Así, el renacimiento de los Juegos Olímpicos Modernos puede ser rastreado en el Congreso de La Sorbona de 1894 en París. Dicho congreso estaba enfocado en otra temática, pero De Coubertin presentó la idea acerca del renacimiento del gran evento antiguo, obteniendo tal éxito en el congreso, que se adoptó la temática del amateurismo en el deporte (Naul et al., 2017). En definitiva, los Juegos Olímpicos poseen un sofisticado componente filosófico promovido por los miembros originales del COI que integra principios éticos, y que, para De Coubertin se convertirían en algo más que un gran evento deportivo, ya que integraría una visión que sería llamada Olimpismo (McLaughlin y Torres, 2012).

Desde entonces ha sido clara la misión del COI en: i) liderar el movimiento olímpico y ii) promover el Olimpismo en todo el mundo (McLaughlin y Torres, 2011). Ambas pueden ser plenamente halladas en los primeros párrafos de la Carta Olímpica, en la que se expresa que el Olimpismo es una filosofía de vida que combina el deporte, la cultura y la educación para crear 
una vida basada en la alegría del esfuerzo; los cuales son encontrados en los principios éticos universales (COI 2019).

De tal forma que el Olimpismo enfatiza que el deporte debe convertirse en una plataforma comprensible, para impulsar sociedades más pacíficas y que deberá incluir de preferencia una formación ética y moral (McLaughlin y Torres, 2011). Esto permitirá promover y construir a mayor escala la sostenibilidad a través de la actividad física, como lenguaje universal en diferentes escenarios sociales.

Por otro lado, los Juegos Olímpicos han evolucionado hacia un espectáculo inmenso y celebrado en diferentes países del mundo. No obstante, este mega evento deportivo ha sido criticado en múltiples ocasiones por diversas razones; aunque, sin embargo, aún siguen siendo ampliamente apreciados y reverenciados en la actualidad (McLaughlin y Torres, 2012). Esto ha generado que, muchos de los críticos y académicos tiendan a alabar solo lo bueno o enfatizar lo malo y esto solo ocultan la realidad de las problemáticas y minimizan la prevención de los peligros que puede enfrentar el Movimiento Olímpico en el futuro (McLaughlin y Torres, 2011).

Por consiguiente, hay una existencia del Ying-Yang — su traducción nos indica la presencia del lado oscuro y el brillante-, tal y como lo contempla la filosofía china sobre dos fuerzas opuestas que se complementan entre sí, y que están presentes en todas las cosas. Un ejemplo de esto en el deporte ha sido que los recientes casos de dopaje en atletas participantes del ciclo Olímpico, los ha llevado a la suspensión temporal de su participación en competencias internacionales.

Entonces, el Movimiento Olímpico tampoco ha sido o será un ejemplo en términos de la promoción de los valores e ideales Olímpicos que este promueve y quizás tampoco lo logrará en próximamente en el futuro (McLaughlin y Torres, 2011). Este argumento, podría visibilizar por qué es importante entender que el deporte no solo promueve actitudes positivas, sino también negativas como resultado de su práctica (Sugden, 1991; Lyras, 2007). Por lo tanto, sería una utopía considerar que el Olimpismo o el deporte por sí mismo causa soluciones mágicas a las problemáticas globales que afectan diferentes dimensiones sociales.

Es importante, también reconocer que el reto fundamental del Olimpismo es surgir de la teoría, para llegar a la práctica y para ello, deberá ser promovido por medio de la educación física, la educación olímpica, el deporte comunitario, iniciativas del deporte para todos y el deporte federado (Lyras, Yiannakis, y Karakalis, 2005; Lyras 2007; Lyras, 2014). En consecuencia, es por medio de las formas arriba mencionadas, que el Olimpismo podría ser implementado en un sistema concreto de actividades, para formar y promover objetivos sociales más amplios hacia el cambio social y la construcción de paz a nivel comunitario.

\subsection{La otra herencia del mundo olímpico: educación física como agente de cambio social}

El antiguo mundo helénico no heredó únicamente la existencia y práctica de los Juegos Olímpicos, sino también, sus valores educativos respecto al balance del cuerpo y la mente. El mismo De Coubertin, era un apasionado educador cuya inspiración como se ha mencionado anteriormente estuvo muy influenciada por esta área de conocimiento. La EF es un elemento 
filosófico que proviene de los antiguos juegos olímpicos y que se integra a los juegos modernos, y que además están presentes como una expresión de la actividad física a lo largo de la historia (Patterson, 2004). A través, de dicha historia han existido diferentes escuelas filosóficas de la EF y diferentes nombres reconocidos como Francois Delsarte o Liselott Diem que incluyeron diferentes características específicas desde su concepción personal de la EF (Culpan y Wigmore, 2010; Weiller Abels y Bridges, 2010).

Por ejemplo, Delsarte enfatizaba el ideal de la estética, que hace énfasis en el desarrollo armónico de la mente, el cuerpo y el espíritu (Weiller Abels y Bridges, 2010). En este sentido, sus ideas pedagógicas encajaban con las promovidas por De Coubertin en sus escritos, en los que señalaba que para que una persona fuese integral era importante que reconocieran la belleza de la práctica de la EF y la educación Olímpica (Girginov y Parry 2005; Culpan y Wigmore, 2010).

Por otro lado, Diem y su colega Grupe, enfatizaban que en la educación de los profesores de EF se precisaba un acercamiento natural para enseñar a los niños a encontrar soluciones a través de sus experiencias de aprendizaje (Weiller Abels y Bridges, 2010) y ambos filosóficamente fueron seguidores de los ideales promovidos por De Coubertin e integraron un componente pedagógico basado en los principios de justicia, solidaridad y paz (Culpan y Wigmore, 2010).

De la misma forma, podemos citar otras filosofías como la de Rudolf Von Laban y Gassmann -profesor de EF Alemán- el primero integraba componentes como las artes, las danza y el segundo integraba virtudes sociales como: la justicia y el respeto mutuo (Culpan y Wigmore, 2010). Estas filosofías han sido pioneras al incluir el deporte, la cultura y la educación como ejes transversales de un currículo de enseñanza hacia el desarrollo integral del ser humano por medio de la EF. Por lo tanto, la EF ha trascendido en el tiempo y De Coubertin lo reconoció como un complemento entendible hacia la promoción de la educación Olímpica.

Los últimos años, diversas investigaciones se han enfocado en identificar los beneficios de la práctica de la EF y el deporte. Por ejemplo, evidencia significativa ha sido catalogada en cuatro áreas: i) física, ii) social, iii) afectiva, iv) cognitiva (Bailey, et al. 2009) y adicional una v) moral (Beedy, 1997). También, esto es reconocido por la Organización Mundial de la Salud (OMS) que argumenta que la actividad física por medio de la EF y el deporte puede convertirse en medio para el desarrollo de las comunidades hacia una mejora de la calidad de vida (ONU, 2015; OMS, 2018).

Sin embargo, para que esto pueda cumplirse por medio de la EF y el deporte, es necesario establecer ambientes adecuados en donde el objetivo principal sea promover estimulaciones humanas (Beedy, 1997). Si estos espacios se diseñan, se pueden promover cambios positivos, disminuir riesgos de problemas sociales como la delincuencia y el uso de drogas (Bailey, et al. 2009). Es decir, se ha demostrado que la EF provee experiencias de aprendizaje a los participantes lo cual les permite construir y reforzar sus habilidades para la resolución de conflictos, autonomía y otras a través de su práctica (Bailey, et al. 2009).

Además, la dimensión moral permite enseñar a los participantes, que el deporte puede tener un resultado positivo o negativo de acuerdo a sus decisiones (Ponciano Nuñez, P, D. 2019). Asimismo, lo moral hace referencia al desarrollo del pensamiento crítico y que forma en los 
participantes una mejor toma de decisiones (Beedy, 1997). Por lo tanto, la EF ha proporcionado avances significativos desde múltiples perspectivas a cerca de sus beneficios y se ha convertido en una herramienta para diferentes organizaciones gubernamentales y no gubernamentales para generar intervenciones deportivas para alcanzar objetivos sociales más amplios (Ponciano Nuñez, P, D. 2019).

\subsection{Deporte para el desarrollo: relaciones olimpicas y programas de desarrollo positivo de la juventud}

El potencial del deporte para contribuir en la educación y el desarrollo integral de niñas, niños y jóvenes ha sido un punto crucial para las diferentes investigaciones que han sido llevadas a cabo desde diferentes perspectivas académicas a través del tiempo. Así en el año 2000, surgió una nueva dimensión conocida como deporte para el desarrollo (DPD) y que fue resultado de la promoción de los Objetivos de Desarrollo del Milenio (ODM) de la ONU (Lyras A 2007; Lyras A 2012; Schulenkorf, Sherry, y Rowe, 2016).

Además, es claro que esta dimensión empezó durante la 54 ed. de la Asamblea General de la ONU, la cual, atrajo nuevas investigaciones a esta área (ONU 2000), incluso se puede identificar mucho antes, remontándose a los antiguos juegos olímpicos y los programas de atención a veteranos heridos durante la Primera Guerra Mundial (Welty Peachey, et al. 2019). Podemos citar también, el interés de profundizar en el uso del deporte más allá de mejorar las habilidades atléticas como tradicionalmente ha sido reconocido. Sin embargo, el DPD ha sido cuestionado debido a la falta de evidencia de los beneficios que dice promover y también por la falta de teoría relacionada, por lo cual se ha sugerido realizar más investigaciones al respecto (Coalter, 2013).

Para responder a esta crítica, en los últimos años diversos autores han sido reconocidos por proporcionar evidencia con teoría significativa en el área del DPD (Lyras A 2007, p. 40; Lyras A 2012, pp. 46-47; Lyras y Welty Peachey, 2011; Lyras y Welty Peachey, 2018; Welty Peachey, Schulenkorf y Spaaij, 2019; Welty Peachey et al., 2019) y se ha asumido por medio de esta evidencia en el cuerpo del conocimiento, que el deporte puede tener un efecto positivo en la salud, el cambio social, construcción de la paz y desarrollo económico de las comunidades, como es mencionado en la definición conceptual de DPD.

Para comprender el funcionamiento de las intervenciones en el terreno que utilizan el DPD, y en este sentido también que sea una teoría que integre dentro de sí misma la esencia del olimpismo. En este caso, una de las teorías más establecidas es la «Teoría del Olimpismo en Sociedad» reconocida en la bibliografía como «Teoría del Deporte para el Desarrollo» (Sport for Development Theory - SFDT), la cual fue elaborada por el Doctor Alexis Lyras de origen chipriota - República de Chipre-, quién argumenta haberla creado ante la falta un marco teórico, por lo cual utilizó la metodología fundamentada, para diseñar y evaluar iniciativas basadas en el uso del Olimpismo.

Todo ello dio como resultado el origen del modelo Doves Olympic Movement Model (DOM) que genera un acercamiento de diferentes teorías interdisciplinarias adaptables al contexto local, para entender bajo qué condiciones el deporte, la educación y la cultura (Olimpismo) promueven cambios personales y sociales para convertirse en un vehículo hacia el 
desarrollo de la paz y la reconciliación (Lyras y Welty Peachey, 2011; Lyras y Welty Peachey, 2018; Marshall y Barry, 2015) El DOM, está inspirado en el uso de los valores Olímpicos orientados a alcanzar en su momento los ODM para promover la aceptación interétnica entre Turcos y Griegos Chipriotas, y es que los ideales olímpicos enfatizan un nivel fraternal del deporte, que puede romper barreras locales, nacionales e internacionales (Sugden, 1991).

La SFDT ofrece cinco componentes que integran un marco teórico para diseñar, implementar y evaluar los resultados de un programa de DPD. Los cinco componentes contienen una serie de elementos que permiten localizar el contenido apropiado de los programas para que los mismos puedan generar cambio social; además, propone que el impacto de los programas debe ser medido en tres niveles: el impacto junto con la sostenibilidad del programa a largo plazo (Lyras y Welty Peachey, 2011; Lyras, 2021).

No obstante, aunque el objetivo de este artículo no es indagar directamente en la SFDT, es necesario mencionarla, ya que sus características científicas y filosóficas parten del Olimpismo y, por medio de ella, transforma la teoría en un modelo práctico. Además, la SFDT ha sido utilizada en múltiples contextos a nivel mundial para evaluar diferentes organizaciones (Lyras y Welty Peachey, 2011; Lyras A, 2012; Marshall y Barry, 2015; Lyras y Welty Peachey, 2018; Welty Peachey, Schulenkorf, y Hill, 2019) y más recientemente por Ponciano Nuñez (2020) a través de un estudio de caso de una fundación juvenil no gubernamental en Colombia, siendo este el primer estudio en América Latina, que utiliza este modelo teórico y práctico validado que posteriormente pretende ser replicado en Guatemala.

\subsection{Programas e iniciativas olímpicas: de la teoría a la práctica en Guatemala}

\subsubsection{Día nacional del deporte para el desarrollo y la paz}

En Guatemala el DPD a incursionado con mucha fortaleza en las instituciones que forman el sistema deportivo nacional, lo cual ha permitido establecer un día para celebrar y promover dicho movimiento. Esto es posible a través de la Ley Nacional para el Desarrollo de la Cultura Física y el Deporte, mediante el Decreto Número 76-97 del Congreso de la República de Guatemala.

Dicha ley, tiene por objeto «regular lo relativo a la coordinación, articulación e interrelación de los sectores institucionales de la educación física, el deporte no federado, la recreación física y el deporte federado para garantizar la práctica de tales actividades físicas como un derecho de todo guatemalteco, creando para el efecto el Consejo Nacional del Deporte, la Educación Física y la Recreación (CONADER)»(Congreso de la República de Guatemala, 2016).

En consecuencia, se promulga el Decreto Número 23-2016 que describe tres resultados en ella: i) Declarar el 6 de abril de cada año «Día Nacional del Deporte para el Desarrollo y la Paz», ii) se enfatiza que CONADER es el responsable de «definir, promover y ejecutar actividades y campañas para conmemorar el Día Nacional del Deporte para el Desarrollo y la Paz en la República de Guatemala» y iii) «las instituciones del deporte, educación física y recreación del Estado deberán proponer e implementar planes, programas y proyectos que aplicados de manera integrada con otras políticas, alianzas y programas especialmente de 
educación, utilicen el deporte como una herramienta promotora de la paz y el desarrollo de la nación» (Congreso de la República de Guatemala, 2016).

La promulgación del Decreto Número 23-2016, ha abierto una oportunidad significativa a nivel nacional, para incluir objetivos de desarrollo sostenible y establecer intervenciones a nivel comunitario hacia la promoción del cambio social y la construcción de la paz, en las diferentes agendas de las instituciones que conforman el CONADER. Suponiendo que realmente las autoridades que en su momento estén a cargo de dichas instituciones comprendan el valor de la educación física y del deporte para alcanzar objetivos sociales más amplios.

Sin embargo, este decreto pudiese también ser utilizado como una base legal que permita establecer programas de capacitación para quienes ejercen los roles y actividades realizadas por ambos sexos de: entrenamiento deportivo, docencia en educación física, promoción y monitoreo deportivo, y liderazgo comunitario; que pudiesen posteriormente, servir como agentes de cambio y beneficiar con personal capacitado a las diferentes instituciones del CONADER y ONG al servicio comunitario.

\subsubsection{Foro de las américas: deporte para el desarrollo y la paz}

El primer Foro de las Américas: Deporte para el Desarrollo y la Paz se llevó a cabo en el mes de abril de 2017, en el Auditorio Mayor del Palacio de los Deportes en la ciudad de Guatemala. El foro se creó como un acto de conmemoración del Día Internacional del Deporte para el Desarrollo y la Paz, y es organizado por el Comité Olímpico Guatemalteco con el apoyo de Solidaridad Olímpica, CONADER y ONU en Guatemala.

El propósito del foro es despertar conciencia en los diversos sectores de la sociedad sobre el poder del deporte para lograr los objetivos de paz. Este es un foro de grandes dimensiones en favor del DPD en la región, aunque es necesario cuestionarse sobre su impacto en la sociedad guatemalteca. Por lo que es recomendable evaluar su contribución a nivel institucional y beneficios para la comunidad para contextualizar las necesidades de las mismas para proponer el deporte como una alternativa para promover el desarrollo sostenible.

\subsubsection{Mi amigo olímpico}

El programa «Mi Amigo Olímpico» (MAO) fue creado por el Comité Olímpico Guatemalteco y el Ministerio de Educación de Guatemala (MINEDUC), y vincula al nivel escolar la educación olímpica dentro del Currículo Nacional Base de educación de Guatemala. En este sentido, Vargas Daetz y Blas (2015) lo definen «como un proyecto ambicioso él cuál se puede hacer realidad conjuntamente con aquellas que comparten el objetivo de construir una Guatemala mejor y más pacifica» (p. 2).

Es un programa que busca integrar diferentes sectores sociales y para que se involucren a utilizar el deporte, la cultura y la educación como un vehículo de cambio social en la juventud guatemalteca. El programa comienza con la visita en el año 2005 de Steve Mester quien fuese Campeón Olímpico y Fundador de Classroom Champions un programa de origen canadiense. Posteriormente, por medio de la cooperación con el MINEDUC el programa se desarrolla actualmente en diferentes escuelas a nivel nacional (1067 sedes), en donde se han capacitado a los directores en la metodología del programa (Vargas Daetz y Blas, 2015). Otra característica 
interesante del programa es el uso de un atleta como mentor para las clases de alumnos participantes durante todo el año lectivo y que, por medio de retos en ocho lecciones, se incluyen valores y habilidades para la vida.

De acuerdo con Vargas Daetz y Blas (2015) «el programa lleva 5 años de funcionamiento y se ha medido el impacto que el mismo tiene en los estudiantes y ha sido muy satisfactorio e inspirador las respuestas recibidas tanto por estudiantes como por maestros y atletas» (p.2). Por medio de la revisión del manual del programa, se evidencia que las evaluaciones son llevadas a cabo de manera cuantitativa y cualitativa por los responsables del mismo. Recientemente, se publicó un artículo adaptado que compara este programa con la versión de Classroom Champions que a través de ciertos indicadores pretende mostrar cómo los atletas pueden ser excelentes modelos a seguir para niños, niñas y maestros participantes del programa de educación olímpica (Blas Fernandez, 2021).

\subsubsection{Fundación Olímpica Guatemalteca}

La Fundación Olímpica Guatemalteca (FUNOG) fue fundada en 1994 por un grupo de dirigentes deportivos con experiencia en temas de deporte y empresariales. El programa es una propuesta de educación física extracurricular, cuyo objetivo es implementar deportes de federaciones deportivas nacionales a niños de áreas marginales para transmitir valores por medio de un programa de educación olímpica (Arimany, 2017). En la actualidad tiene intervenciones comunitarias en cinco zonas de la ciudad de Guatemala y dos departamentos del país. De manera resumida, su propósito es contribuir a la transformación de niños jóvenes que viven en zonas de riesgo por medio del deporte.

Recientemente, en el año 2018, se llevó a cabo un estudio significativo de evaluación de impacto en el desarrollo de habilidades para la vida y niveles de agresión de participantes del programa Hoodlinks de la Fundación Olímpica Guatemalteca, la cual está estructurado en base de los valores Olímpicos y el deporte para enseñar habilidades para la vida (Mandigo, Corlett y Holt, et al., 2018). Este estudio encaja con estudios anteriores que indican que los valores Olímpicos pueden ser enseñados por medio de actividades planificadas y que pueden llegar a tener un impacto en el desarrollo integral de niños y jóvenes participantes de los programas (Lyras, 2007; Lyras, 2012).

También, los hallazgos del estudio resaltan la importancia de implementar programas en vecindarios o barrios donde las condiciones de vulnerabilidad social son muy altas, por lo que proporcionar espacios seguros es primordial para que aprovechen su tiempo libre y se minimizan las posibilidades de integrarse en la violencia y actividades criminales (Lyras, 2012). Por lo tanto, es necesario continuar investigando cómo se pueden multiplicar programas similares en Guatemala partiendo de los resultados positivos de este estudio realizado al programa Hoodlinks (Mandigo, Corlett y Holt, et al. 2018).

Sin embargo, el estudio carece de un análisis de las estructuras organizacionales y administrativas que han hecho que la FUNOG sea considerada una ONG para el desarrollo juvenil a través del deporte exitosa, pero es necesario reconocer que este estudio es pionero desde la perspectiva del DPD en Guatemala y Centro América. 


\section{ANALISIS Y DISCUSIÓN DE RESULTADOS}

El uso del análisis documental utilizado como metodología demuestra que en las últimas dos décadas ha existido un aumento en el interés de las agencias gubernamentales y no gubernamentales en el uso del deporte para alcanzar objetivos sociales más amplios. En este sentido, Lyras y Welty Peachey (2011) hacen referencia a la necesidad de centrar estudios sobre las múltiples intervenciones desarrolladas bajo la creencia que el deporte puede tener un impacto en las personas o comunidades. Por otro lado, históricamente una de estas organizaciones es el COI quienes han propuesto el Movimiento Olímpico como una plataforma comprensible para la promoción de la alfabetización motora y una herramienta para el fomento del cambio social, la construcción de la paz y objetivos de desarrollo sostenible. Por lo consiguiente, el MOI cumple con las dos perspectivas fundamentales de ser un proceso educativo y realizar un gran evento deportivo en donde los participantes aprendan y posteriormente compartan los valores olímpicos (Naul et al, 2017).

Sin embargo, los resultados demuestran como el deporte puede ser un medio para resultados positivos y negativos en dependencia de como este sea practicado (Sugden, 1991; Lyras, 2007, 2021). En este caso, el Movimiento Olímpico tampoco ha sido históricamente un gran ejemplo de cumplir con éxito los valores e ideales olímpicos que el COI promueve (McLaughlin y Torres, 2011). Por esta razón, es necesario proponer el uso de teorías ampliamente reconocidas en el cuerpo del conocimiento del DPD y que han progresivamente contribuido a cerrar la brecha de la falta de un marco teórico que permita diseñar, implementar y evaluar iniciativas que integren el uso de la filosofía del olimpismo en la educación física y el deporte para alcanzar objetivos sociales más amplios. Por ejemplo, la teoría más reconocida y utilizada en múltiples contextos integrando múltiples teorías interdisciplinares es la "Teoría del Deporte para el Desarrollo" (Sport for Development Theory -SFDT-) (Welty Peachey, et al. 2019).

La SFDT está fundamentada en el uso de la filosofía olímpica para tributar a alcanzar los objetivos del desarrollo del milenio. Esta teoría sugiere que las experiencias deportivas, pueden impactar de forma positiva, solo si ciertos elementos son considerados como catalizadores para optimizar a largo plazo y de forma sostenible resultados educativos y sociales (Lyras y Welty Peachey, 2011). Además, Sugden (1991) considerado el pionero de los estudios en DPD claramente mencionaba que los ideales olímpicos crean espacios significativos que podían romper barreras nacionales e internacionales. Por esta razón, Lyras y Welty Peachey (2011; 2018) argumenta en su teoría ampliamente reconocida está concebida con base a los tres componentes fundamentales del olimpismo que son el deporte, ii) la educación y iii) la cultura.

Además, incluye dos componentes más relacionados a i) evaluación del impacto y ii) elementos organizaciones y administrativos que permiten a las organizaciones que trabajan con el DPD tener resultados sostenibles (Lyras y Welty Peachey, 2011; Marshall y Barry 2015). También, la revisión de la bibliografía arrojó como resultado que recientemente el único caso a nivel del Sur Global como se le conoce a Centro América y Sudamérica que utiliza una teoría reconocida en el campo del DPD, fundamentada en el olimpismo y que podría ser replicada en Guatemala es el estudio realizado por Ponciano Nuñez (2020) en el que se demuestran los componentes organizaciones y administrativos de una fundación juvenil que utiliza el deporte para el desarrollo positivo de la juventud en Colombia por medio de la aplicación de la SFDT. 
En este sentido, se ha identificado el panorama de iniciativas para el desarrollo del DPD en Guatemala cuyo origen está fundamentado en el olimpismo. La primera es la promulgación del Decreto 23-2016 que hace un llamado a la necesidad de proponer e implementar planes, programas y proyectos en el que el deporte sea utilizado como un vehículo para la promoción de la paz y el desarrollo de la nación (Congreso de la República de Guatemala, 2016). No obstante, es de recalcar la necesidad que estas iniciativas se han diseñados sobre marcos teóricos reconocidos y probados que permitan maximizar la posibilidad de alcanzar los resultados deseados de las mismas permitiendo contribuir al alcance de objetivos sociales de desarrollo comunitario de las poblaciones más vulnerables. La segunda, El Foro de Las Américas es llamado a ser el espacio en el que los diferentes sectores de la sociedad reconozcan y apoyen el uso del deporte como una alternativa para alcanzar los ODS. Sin embargo, no hay evidencia científica que permita reconocer si verdaderamente este evento de grandes dimensiones ha tenido un impacto significativo más allá de ser un espacio de socialización y promoción de políticas públicas.

La tercera, el programa MAO está vinculado al sistema educativo escolar utilizando el concepto de la educación olímpica y cuyo objetivo es construir una Guatemala mejor y más pacífica (Daetz y Blas, 2015). La revisión de su manual evidencia que llevan a cabo evaluaciones cuantitativas y cualitativas a los participantes para medir el impacto de su intervención resultado de la aplicación de sus manuales. Así, lo explica Blas Fernández, J, D (2021) en su estudio publicado recientemente que compara el resultado del programa Classroom Champions y MAO a través de sus informes de organizaciones. Sin embargo, el estudio, aunque indica discutir datos cualitativos, muestra resultados del impacto sobre porcentajes numéricos producto de la encuesta cerrada y no discute conforme a la evidencia de un análisis de datos cualitativos de evidencia entorno de declaraciones de los participantes de las organizaciones que permita profundizar en las creencias y experiencias en torno a que los atletas tienen un impacto significativo producto de su interacción temporal en el grupo objetivo.

Además, resalta que los atletas pueden tener un impacto mucho mayor en base a la admiración que los individuos tienen en ellos, más que un maestro u otro tipo de facilitador, pero luego acertadamente hace un llamado al trabajo inter grupal de atletas, profesores y familias para que la enseñanza de valores se consolide en casa (Blas Fernández, J, D, 2021). En este sentido, es importante resaltar que los atletas o voluntarios pueden tener un rol importante en el proceso, más no el más significativo, ya que la mayoría de ellos no poseen una preparación teórica y práctica para ejecutar un proceso pedagógico hacia el aprendizaje de valores adecuado a las características de los participantes y dependen de estructuras validadas por educadores, psicólogos y antropólogos para conducir la intervención.

Finalmente, citaremos la iniciativa del programa FUNOG cuyo objetivo es contribuir a la transformación de niños y jóvenes que viven en zonas de riesgo por medio del deporte extracurricular y la clase de educación física. Por el momento este programa es el único que cuenta con un estudio científico que demuestra la necesidad de contar espacios seguros en donde los jóvenes en condiciones vulnerables aprovechen su tiempo libre y disminuyan sus posibilidades de integrarse en la violencia y el crimen organizado (Mandigo, Corlett y Holt, et al. 2018). En este sentido, FUNOG actualmente no forma parte del sistema educativo nacional guatemalteco, pero a diferencia de $\mathrm{MAO}$, tienen una intervención permanente en las comunidades. 
Aunque, este supone un inicio positivo para iluminar como el DPD se utiliza en Guatemala, carece de un análisis profundo de sus estructuras organizacionales y administrativas que han hecho de FUNOG ser considerada una ONG exitosa en el uso del deporte para el desarrollo positivo de la juventud.

\section{CONCLUSIONES Y REFLEXIONES}

El estudio documental ofrece un marco histórico que conceptualiza cómo el Olimpismo ha sido utilizado desde la antigua civilización griega a tiempos modernos como vehículo de cambio social y desarrollo comunitario. Además, se alinea a la necesidad de reconocer la actual división olímpica entre el ideal abstracto y la realidad actual de hacer un llamado al uso de evidencia científica sobre cómo utilizar el Olimpismo para el bien de la humanidad (Lyras, 2021). Esto ilumina cómo la teoría debe transitar a la práctica utilizando las tendencias modernas de programación de intervenciones comunitarias para convertir la educación física y el deporte en un catalizador de la formación integral de ciudadanos como se añoraba en los antiguos Juegos Olímpicos helénicos.

Por otra parte, el estudio demuestra que la filosofía del Olimpismo inmersa en programas del DPD puede ser un vehículo para el enriquecimiento cultural en el que la educación física puede ser un medio efectivo para transformar instintos violentos y disminuir la agresividad que dará como resultado un aumento significativo en el potencial de creatividad, formación de valores y virtudes en los participantes. También, el reconocer el panorama de iniciativas del DPD en eventos o intervenciones de programas que infunden el Olimpismo en Guatemala permiten iluminar que las mismas se enfocan en unificar individuos de diferentes antecedentes sociales por medio de la educación, la actividad física y artística que persigue la excelencia y la innovación.

Sin embargo, esto incrementa la necesidad de reconocer la importancia de la elaboración de un diseño, implementación y evaluación rigurosa de las intervenciones sociales por medio de la educación física y el deporte. Para ello, este estudio integra la propuesta de una teoría enfocada en el deporte para el desarrollo y la paz que existe desde la primicia de alcanzar los objetivos del desarrollo del milenio y que en la actualidad contribuye proporcionando componentes efectivos basados en teorías interdisciplinarias pertenecientes a la educación, la cultura, el deporte, la administración y la psicología para que estas experiencias incrementen el cuerpo del conocimiento limitado producido actualmente en América Latina. Además, se hace un llamado a otros académicos de Centro América y específicamente de Guatemala en las ciencias de la educación física y el deporte para involucrarse en contribuir al desarrollo del DPD y fortalecer la importancia del uso del deporte como una herramienta de cambio social y construcción de la paz.

Por otro lado, a través de este estudio se evidencia como FUNOG se ha convertido en un faro en la región respecto al uso de la educación física y la práctica de deportes reconocidos en el sistema deportivo nacional de Guatemala, que abre oportunidades deportivas para niños, niñas y jóvenes, que a la vez aprenden habilidades para la vida. Esto evidencia aún más cómo el deporte de alto rendimiento evade su responsabilidad social de promover el desarrollo comunitario (Ponciano Nuñez y Lyras, 2018). Además, MAO se convierte en un programa que también puede tener un impacto significativo por medio de su intervención basada en el uso de atletas 
como modelos de rol y complementando las lecciones con apoyo de los maestros y padres de familia. Sin embargo, ambos programas aún carecen de fundamentación científica basada en modelos teóricos sólidos del DPD.

Así, Guatemala tiene la oportunidad de convertirse en un ejemplo para la región centroamericana, en la cual las federaciones y asociaciones deportivas afiliadas al Movimiento Olímpico, reconozcan e integren políticas internas para aportar hacia al avance la consecución de los índices de desarrollo humano a través de la EF y el deporte. Por lo tanto, las instituciones que integran el CONADER a través del uso de la EF y el deporte, para liderar avances significativos en DPD y son parte de una de las plataformas más sostenibles como lo es el Movimiento Olímpico. De esta manera, el deporte podría convertirse en un medio para romper barreras de clases sociales y promover intercambios interétnicos en comunidades en donde aún existen formas conflictivas de relacionamiento.

Sin embargo, no se debe de obviar que Guatemala cuenta con el "Foro de las Américas: Deporte para el Desarrollo y la Paz" uno de los eventos más importantes a nivel regional respecto al tema de DPD. Aunque, hasta el día de hoy dicho evento ha tenido tres ediciones y aun se desconocen los impactos que el mismo ha generado a nivel micro (nacionales), meso (regionales) y macro (globales) en beneficio de la comunidades guatemaltecas. En este sentido, para cerrar la brecha de desconocimiento de los beneficios y oportunidades que han sido generadas por esta iniciativa un sistema más efectivo de evaluación abierta, debería ser incorporado por las organizaciones encargadas del evento.

Además, las instituciones que gobiernan el sistema deportivo en Guatemala deben diseñar e implementen programas basados en evidencia científica para intervenir en las comunidades vulnerables y cuyos pilares fundamentales se enfoquen a la formación integral, la ética y las relaciones de convivencia hacia una sociedad más pacifica. Para ello, Guatemala cuenta con estructuras organizacionales y legales para posicionar la EF, y el deporte como una alternativa realista para integrar dentro de ellas el concepto del Olimpismo. También, esto es respaldado por los expertos al recomendar que esta filosofía de preferencia debe integrarse por medio de la EF (Culpan 2018). Además, para ello será necesario formar personal capacitado dentro de las instituciones con habilidades intelectuales y prácticas, que permita formar nuevos modelos de rol para las futuras generaciones y que puedan innovar fuera de los modelos pedagógicos tradicionales de la EF y el deporte.

\section{RECOMENDACIONES}

En base a los resultados de este estudio se recomienda fomentar un espíritu en torno a un pensamiento crítico que reconozca que la educación física y el deporte pueden tener un efecto positivo y negativo al no ser consideradas las condiciones necesarias que han sido halladas en base a investigaciones científicas de impacto realizadas en los últimos veinte años.

Así mismo, se convierte en necesario recomendar que el cuerpo del conocimiento respecto al DPD pueda ser actualizado y accesible a todos los interesados evitando de esta manera acrecentar la brecha en la información entorno al uso del deporte para alcanzar objetivos sociales más amplios. Finalmente, se recomienda promover estudios experimentales adaptando marcos teóricos internacionales al contexto guatemalteco y que permita medir el impacto de los 
programas que utilizan la educación física y el deporte para promover el cambio social.

\section{BIBLIOGRAFIA Y FUENTES}

Arimany, A. (2017). Enfoques y Aplicaciones de la Educación Olímpica. Citius, Altius, Fortius, 10 (1), 1-17. https://doi.org/10.15366/citius2017.10.1.001

Bailey, R., Armour, K., Kirk, D., Jess, M., Pickup, I., \& Sandford, R. (2009, February 19). The educational benefits claimed for physical education and school sport: an academic review. Research Papers in Education, 24(1), 1-27. https://doi.org/10.1080/02671520701809817

Blas Fernández, J.D. (2021). La Importancia de los Atletas como Modelos a Seguir en la Enseñanza de la Educación Olímpica. Citius, Altius, Fortius. Humanismo, Sociedad y deporte. Investigaciones y ensayos. 14 (1), 9-14. https://doi.org/10.15366/citus2021.14.1.002

Beedy, J. (1997). Sports Plus Positive Learning Using Sports: Developing Youth Sports Programs that Teach Positive Values. Project Adventure, Inc.

Clastres, P. (2010). Playing with Greece. Pierre de Coubertin and the Motherland of Humanities and Olympics. Histoire@Politique.Politique, culture, société(12), 1-14. Retrieved from www.histoire-politique.fr. https://doi.org/10.3917/hp.012.0009

Coalter, F. (2013). Sport for development: what game are we playing? Routledge. https://doi.org/10.4324/9780203861257

Comité Olimpico Internacional. (2019). Carta Olimpica. DidWeDo S.a.r.1. .

Congreso de la República de Guatemala . (2016). Decreto Número 23-2016. Centro Nacional de Análisis y Documentación Judicial .

Culpan, I. (2018). Olympism, Physical Education and Critical Pedagogy. European Physical Education Review, 25(3), 851-856. https://doi.org/10.1177/1356336X18782560

Culpan, I., \& Wigmore, S. (2010). The Delivery of Olympism Education within a Physical Education Context Drawing on Critical Pedagogy. International Journal of Sport and Health Science, 67-76. https://doi.org/10.5432/ijshs.20090028

Girginov, V., \& Parry, J. (2005). The Olympic Games Explained (1era ed.). Routledge.

Koulouri, C. (2006). The First Modern Olympic Games at Athens. European Context in European Studies, 5.

Lamoneda, J. (2017). Intervention to educate adolescents in a culture of peace through sports in social exclusion contexts. E-Balonmano.com: Journal of Sport Science, 225-236. 
Lyras , A., \& Welty Peachey, J. (2011, May 14). Integrating sport-for-development theory and praxis. Sport Management Review, 311-326. https://doi.org/10.1016/j.smr.2011.05.006

Lyras , A., Yiannakis , A., \& Karakalis, M. (2005). Sports as a Medium for Personal and Social Change. Sports as a Medium for Personal and Social Change.

Lyras, A. (2007). Characteristics and Psycho-Social Impacts of an Inter-Ethnic Educational Sport Initiative on Greek and Turkish Cypriot Youth. University of Connecticut.

Lyras, A. (2009). Proceedings from the 17th Annual European Sport Management Conference: Sport for peace and development theory. Amsterdam, Netherlands .

Lyras, A. (2012). Olympism in Practice: Psychosocial Impacts of an Educational Sport Initiative on Greek and Turkish Cypriot Youth. The ICHPER-SD Jounal of Research in Health, Physical Education, Recreation, Sport \& Dance, 7, 46-54.

Lyras, A. (2014). Olympic Education in Practice: Educational Components of a Sports for Peacebuilding Intervention. En N. Müler y D. Chatziefstathiou, Olympism, Olympic education and learning legacies, (pp. 245-258). Cambridge Scholars Publishing.

Lyras, A., \& Welty Peachey, J. (2018). The Conception, Development, and Application of SportFor-Development Theory. In J. S. de George B. Cunningham, Routledge Handbook of Theory in Sport Management, (pp. 131-136). Routledge. https://doi.org/10.4324/9781315753461-11

Lyras, A. (2021, Junio). Olympism for Humanity Theory and Praxis: A Call for Peace and Democracy Champions of Change. Peace in Conflict: Journal of Peace Psychology. Advance online publication. https://doi.org/10.1037/pac0000498

Mandigo , J., Corlett , J., Holt, N., van Ingen, C., Geisler, G., MacDonald, D., \& Higgs, C. (2018). The impact of the Hoodlinks Programme on developing life skills and preventing youth violence in Guatemala City. Journal of Sport for Development, 6(11), 21-37.

Marshall, S., \& Barry, P. (2015). Community Sport for Development: Perceptions From Practica in Southern Africa. Journal of Sport Management, 109-121. https://doi.org/10.1123/JSM.2012-0301

McLaughlin, D., \& Torres, C. (2012). More Than Games Olympism as A Moral Approach to Sport. University Press of Kentucky.

McLaughling , D., \& Torres , C. (2011). A Moral Justification for a More Inclusive Olympic Program. Olympika: The International Journal of Olympic Studies, 55-78.

Naul, R., Binder, D., Rychtecky, A., \& Culpan, I. (2017). Olympic Education. Routledge. https://doi.org/10.4324/9780203131510

Organización de Naciones Unidas. (2000, Enero 18). Building a peaceful and better world through sport and the Olympic ideal. UN Publishing. 
Organización de Naciones Unidas. (2015). Transforming our world: the 2030 Agenda for Sustainable Development. UN Publishing .

Organización Mundial de la Salud. (2018). Global Action Plan on Physical Activity 2018-2030: More Active People for a Healthier World. OMS.

Patterson, J. (2004, September). Why Teach Physical Education History? Journal of Physical Education, Recreation \& Dance, 75(7), 29-32. https://doi.org/10.1080/07303084.2004.10607271

Peachey, J.W., Schulenkorf, N., \& Spaaij, R. (2019). Sport for Social Change: Briding the Theory-Practice Divide. Journal of Sport Management, 33(5), 361-365. https://doi.org/10.1123/jsm.2019-0291

Peachey, J. W., \& Cohen, A. (2016). Research Partnerships in Sport for Development and Peace: Challenges, Barriers, and Strategies. Journal of Sport Management, 30(3), 282-297. https://doi.org/10.1123/jsm.2019-0291

Peachey, J. W., Schulenkorf , N., \& Hill, P. (2019). Sport-For-Development: A Comprehensive Analysis of Theoretical and Conceptual Advancements. Sport Management Review, 23(5), 783-796. https://doi.org/10.1016/j.smr.2019.11.002

Ponciano Nuñez, P. D., \& Lyras, A. (2018, October). Basketball, Innovation and Change Agency: Historical Overview and Current Landscape. Sport and Olympic-Paralympic Studies Journal, 3(1), 155-163.

Ponciano Nuñez, P. D., \& Lyras, A. (2018). Theory and Practice of Olympism in Society: A Case Study Analysis of Tiempo De Juego a Youth Development Foundation in Colombia. Sport and Olympic-Paralympic Studies Journal, 3(1), 227-236.

Ponciano Nuñez, P. D. (2020). Peacebuilding Through Olympism: A Case Study Analysis of a Youth Development Foundation in Colombia. Olympic Studies: $26^{\text {th }}$ International Seminar on Olympic Studies for Postgraduate Students Proceedings, pp.50-61

Rato Barrio, M. (2009). La Actividad Física y el Deporte como Herramienta para Promover el Interculturalismo en Contextos Postbélicos, en el Marco de la Cooperación para el Desarrollo. Un proyecto realizado en Guatemala (CentroAmérica) [Tesis de Doctorado, Universidad Politécnica de Madrid]. Archivo Digital UPM. https://oa.upm.es/1674/

Sakoulas, T. (2016). Ancient-greece.org. Recuperado el 2 de mayo de 2018 de http://ancientgreece.org/culture/olympic-games.html

Schulenkorf, N., Sherry , E., \& Rowe , K. (2016). Sport for Development: An Integrated Literature Review. Journal of Sport Management, 30(1), 22-39. https://doi.org/10.1123/jsm.2014-0263 
Sugden, J. (1991). Belfast united: Encouraging cross-community through sport in Northern Ireland. Journal of Sport and Social Issues, 15(1), 59-80. https://doi.org/10.1177/019372359101500104

Tancara Q, Constantino. (1993). La investigación documental. Temas Sociales, (17), 91-106. Recuperado en 04 de julio de 2021 de: http://www.scielo.org.bo/scielo.php?script=sci_arttext\&pid=S004029151993000100008 $\underline{\text { \&lng}}=\mathrm{es} \& \mathrm{t} \operatorname{lng}=\mathrm{es}$.

Teetzel, S. J. (2012). Optimizing Olympic education: a comprehensive approach to understanding and teaching the philosophy of Olympism. Educational Review, 64(3), 317-332. https://doi.org/10.1080/00131911.2012.688729

Vargas Daetz, S., \& Blas , J. (2015). Guía Didáctica para el Desarrollo de Valores Olímpicos en el Aula. Guatemala: Academia Olimpica Nacional de Guatemala.

Weiller Abels , K., \& Bridges, J. (2010). Teaching Movement Education: Foundations for Active Lifestyles. Human Kinetics Publishers. https://doi.org/10.5040/9781718208995 